\title{
Non-invasive genetic study and population monitoring of the brown bear (Ursus arctos) (Mammalia: Ursidae) in Kastoria region - Greece
}

\author{
Dimitris Tsaparis $^{\mathrm{a}, \mathrm{c}}$, Nikoleta Karaiskou ${ }^{\mathrm{b} *}$, Yorgos Mertzanis ${ }^{\mathrm{a}}$ \\ and Alexander Triantafyllidis ${ }^{\mathrm{b}}$ \\ ${ }^{a}$ NGO "Callisto", Wildlife and Nature Conservation Society, Thessaloniki, Greece; ${ }^{b}$ Department \\ of Genetics, Development and Molecular Biology, School of Biology, Aristotle University of \\ Thessaloniki, Thessaloniki, Greece; ${ }^{c}$ Institute of Marine Biology, Biotechnology and \\ Aquaculture, Hellenic Centre for Marine Research (HCMR), Heraklion, Greece
}

(Received 8 November 2012; accepted 15 December 2013; first published online 4 April 2014)

\begin{abstract}
The brown bear (Ursus arctos) in Greece is considered endangered but little is known about the genetic status and the exact size of local populations. Noninvasive genetic sampling was used in this study to investigate the genetic diversity and genetic structure of the brown bear population in the Kastoria region (northwest Macedonia, Greece) and to estimate its population size. Estimation of demographic parameters was based on innovative, well-evaluated methods that can provide estimates from a single sampling session. DNA was extracted from hairs, scat and blood samples and subsequent amplification of 10 microsatellite loci allowed the identification of a minimum number of 75 living bears in the study area while the mark-recapture-based analysis resulted in a point estimation of 219 individuals. Relatively high diversity values, lack of heterozygosity deficiency as well as estimated effective population size, support the Kastoria bear population having good conservation status.
\end{abstract}

Keywords: non-invasive genetic sampling; population status; brown bear; Greece

\section{Introduction}

The brown bear (Ursus arctos), the largest carnivore in Europe, has suffered severe population bottlenecks in the past, mainly because of human persecution and habitat degradation, resulting in shrinkage of both its geographical range and abundance. Despite these demographic and habitat pressures, brown bear populations in Greece have managed to survive and the population appears to be stable at around 190-400 individuals, which are split into two independent populations (Mertzanis et al. 2009a; Karamanlidis 2011). The most significant population, in the western part of the country, is part of the Dinaric-Pindos biological population (one of the largest in Europe estimated at 2800 individuals), and the smaller one in the northeastern part of the country is part of the Eastern Balkan population (Linnell et al. 2007). Significant conservation actions and measures during the last two decades, mainly under the LIFE Programme, seem to have substantially contributed to bear population recovery in Greece. Recent re-colonization of areas of historical distribution clearly indicate positive population trends at a local scale (Mertzanis et al. 2009b). Nevertheless,

*Corresponding author. Email: nikolbio@bio.auth.gr

Dimitris Tsaparis and Nikoleta Karaiskou contributed equally to this study 
the brown bear is still considered endangered in Greece, mainly because of humanrelated threats such as poaching, traffic fatalities, or habitat fragmentation due to large infrastructure development, which continues to affect its survival potential (Mertzanis et al. 2009a).

Census population size $\left(N_{c}\right)$, effective population size $\left(N_{e}\right)$ and genetic diversity are significant parameters to assess the population status. It is well known that small populations are more vulnerable to demographic and environmental stochasticity, genetic drift and inbreeding, which increase the probability of extinction (Soulé 1987). Reliable estimations of population size and evaluation of the genetic status are crucial for conservation planning and the proper management of endangered species or populations. Despite the conservation status and the necessity for targeted management projects, very few systematic studies have been conducted so far at a local scale in Greece for bear populations (e.g. Mertzanis 1994; Karamanlidis et al. 2012).

The bear population of the Kastoria region is considered to be a significant part of the larger and geographically more extended bear population of the Pindos mountain range in western Greece. During recent years, increased bear-human interference incidents in this region are probably related to an increase in bear population density, and have multiplied bear-human conflict situations. Moreover, the recent construction of the Egnatia highway segment "Siatista-Krystallopigi" (KA45) added a new threat to the survival of the indigenous bear population because of numerous lethal bear-vehicle collisions. Lack of appropriate mitigation structures, as well as the existence of a non-bearproof highway fence, are considered to be the main reasons for these accidents. These problems combined with a lack of knowledge on basic bear population parameters in Kastoria have made the systematic monitoring and evaluation of its conservation status a necessity.

Bears are difficult to monitor because they are elusive, solitary and occur at low densities. Non-invasive genetic sampling (NGS) has been proposed as a reliable alternative sampling method not only for the genetic study of rare or cryptic animal species but for the estimation of their abundance as well (Kohn and Wayne 1997; Kohn et al. 1999; Bellemain et al. 2005; Waits and Paetkau 2005; Luikart et al. 2010). The main advantage of NGS is that no capturing or handling of animals is required. Although at first there were significant concerns about the genotyping reliability of NGS and the inflating effect that genotyping errors may have on DNA-based abundance estimates (Taberlet et al. 1996; Gagneux et al. 1997; Creel et al. 2003; McKelvey and Schwartz 2004), numerous studies have shown that NGS can provide reliable and accurate information (e.g. Lathuillière et al. 2001; Waits and Paetkau 2005; Sawaya et al. 2011). As a result, most of the recent publications concerning ecology, demography, population genetics or phylogeography of bears have incorporated NGS methods (Gervasi et al. 2008; Pérez et al. 2009; De Barba et al. 2010a; Sawaya et al. 2012; Schregel et al. 2012). In addition, Karamanlidis et al. (2010, 2012) tested the efficiency of implementation and reliability of these methods on brown bear populations in Greece and proposed specific protocols.

The purpose of this study, which is part of an ongoing LIFE project (LIFE09 NAT/GR/000333-Action A3), was twofold: (1) to estimate the genetic diversity and describe the genetic structure of the bear population in the Kastoria region, and (2) to estimate the population size (census and effective) based on NGS and genetic markrecapture data. The results of this study are expected to have direct implications in the management of bear populations in the study area while this paper can assist 
conservation efforts for the species in Greece, providing methodological and practical guidelines for larger-scale monitoring programmes based on NGS in the future.

\section{Material and methods}

\section{Study area}

The study area is located in northwestern Greece and to a great extent overlaps with the prefectural unit of Kastoria (Figure 1). Large parts of the Gramos and Voio mountains, which belong to the northwestern Pindos range, and the valleys of the Sarantaporos and Aliakmon rivers, are included. The study area is delineated westwards by the Sarantaporos river, eastwards by the Vernon and Askio mountains, southwards by the Aliakmonas river in the Kozani prefectural unit and northwards by the border with Albania and the prefecture of Florina. It covers $1720 \mathrm{~km}^{2}$ at altitudes that range between 400 and $2520 \mathrm{~m}$ above sea level. The study area is covered by dense forests, partially forested areas, grasslands and cultivations. Forest vegetation is composed of black pine (Pinus nigra), oak (Quercus sp.) and beech (Fagus sp.) whereas cultivations comprise corn fields, wheat fields and orchards. A large part of the study area is characterized by medium- to high-density human settlements (villages of 50-100 and > 500 inhabitants). A high-density road network $\left(1.5 \mathrm{~km} / \mathrm{km}^{2}\right)$ supporting human activities, the recent construction and operation of highway KA45, and a relatively high level of hunting pressure are among the humanrelated disturbance and mortality factors.

\section{Sampling}

Recent non-invasive genetic studies of small bear populations have shown that the optimal sampling strategy should combine systematic hair trapping and opportunistic sampling of faeces and hairs, because the pooled data allow increased bear identification possibilities and they are suitable for population size estimation (Gervasi et al. 2008; Pérez et al. 2009; De Barba et al. 2010b).

Hairtraps on power poles are one of the sampling procedures that have been suggested for non-invasive genetic studies of brown bears and they have been effectively implemented in Greece (Karamanlidis 2008; Karamanlidis et al. 2010). A permanent sampling network of 110 hairtraps was installed in the study area and was revisited monthly from July to December 2011. The initial selection of the poles was made after inspection of the local power pole network for recent bear signs (claw marks, hairs or mud). All hairs found on one barb of the barbed wire on a given power pole were considered as one sample and stored dry with silica gel at room temperature in a paper envelope. In total, 129 hair samples were collected.

In addition, 42 hair samples were collected with barbed wire attached on the fence of highway KA45. The hairtraps in that case were placed on both sides of the highway - focusing on the spots where bears could easily clear the fence - and revisited on a regular (every 2-3 days) basis for 2 months (October and November 2011).

To further increase sampling size and coverage, we included 46 faecal samples opportunistically collected during regular field surveys from July to December 2011. For species producing large faeces (i.e. brown bear), it is generally not necessary to 


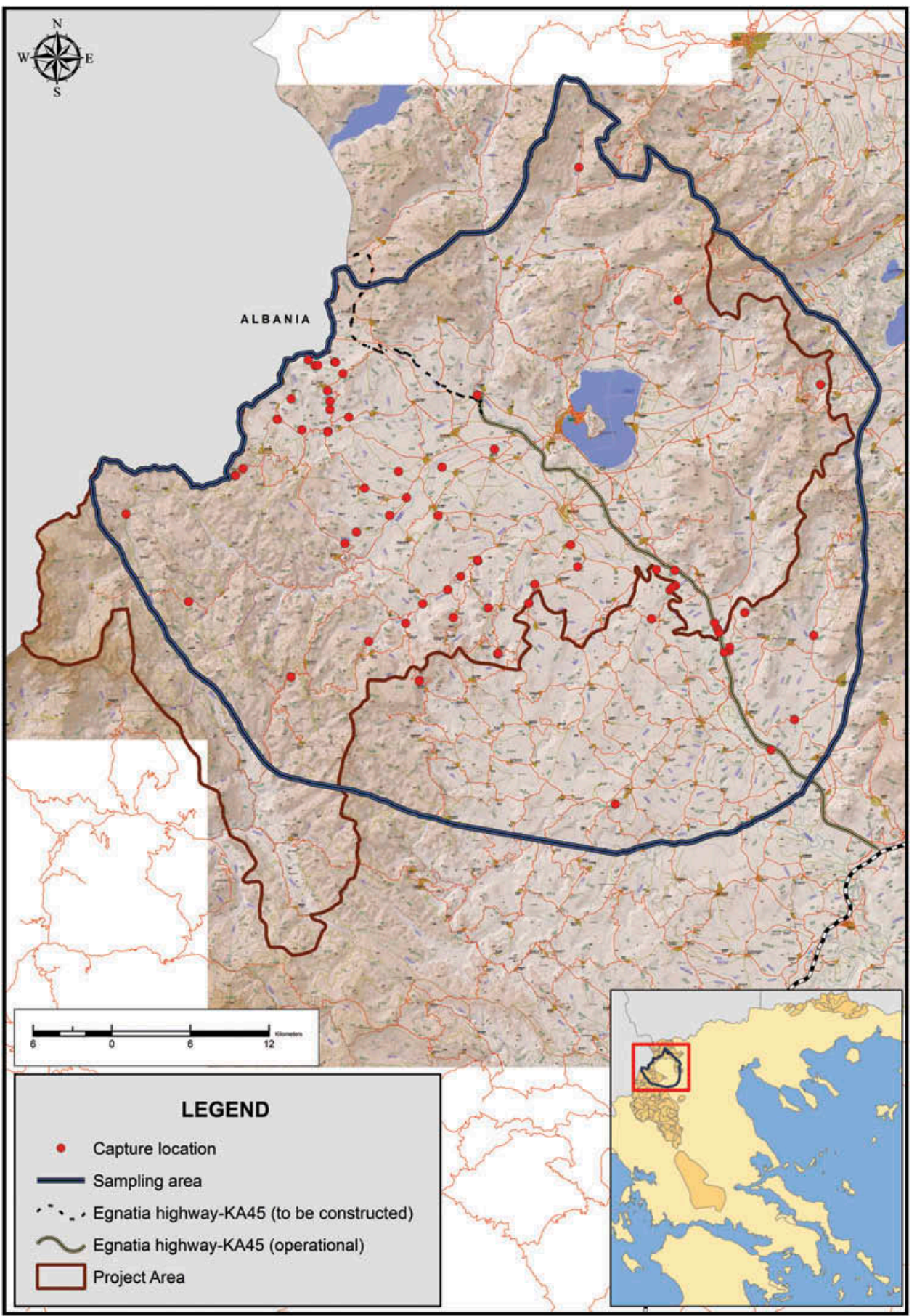

Figure 1. The study area in Kastoria region and capture locations (red dots) for the 75 living bears. 
collect the whole scat and hence a subsample of it is often taken in the field. To maximize DNA quality, the outside portion of bear scats was preferably collected according to Stenglein et al. (2010). Faecal samples were stored in plastic tubes containing absolute ethanol and preserved at $-20^{\circ} \mathrm{C}$.

Fifteen blood samples were also included in the analysis. These samples came either from bears that were victims of vehicle collisions (nine samples) or from live bears trapped for telemetry purposes (six samples). Blood samples were stored in collecting tubes at $-20^{\circ} \mathrm{C}$.

\section{Molecular analysis}

\section{DNA extraction}

Generally, 2-12 guard hairs per sample were used for DNA extraction. Under a stereoscope hair roots were cut and transferred to a 1.5-ml tube. DNA was extracted from hair roots and blood samples using the QIAmp Mini Kit of Qiagen (Hilden, Germany) following the manufacturer's instructions. For scat samples, a QIAmp DNA Stool Mini Kit from Qiagen was used with slight modifications of the manufacturer's protocol (available on demand). A small quantity of faecal material (180 $220 \mathrm{mg}$ ) was scraped off the outer part of each sample using sterile spoons and was left to dry under a fume hood. All extractions from faecal samples took place in a separate facility to avoid contamination. In addition, extraction-negative controls were used. The low quantity of DNA obtained from hair and faecal samples does not allow for direct testing of successful extraction in agarose gel. For this reason, extracted samples were initially amplified with one pair of the selected microsatellite loci and then the polymerase chain reaction (PCR) product was visualized by agarose gel electrophoresis.

\section{Marker amplification}

Ten brown bear microsatellite loci were used in the present study: G1A, G10L, G10P, G10C, G1D (Paetkau et al. 1995), Mu26, Mu59 (Taberlet et al. 1997), G10H (Paetkau et al. 1998), G10X (Taberlet et al. 1997; Paetkau et al. 1998) and Mu50 (Bellemain and Taberlet 2004). The amplified loci were combined into three Multiplex PCR to reduce the amount of DNA, the total number of reactions per experiment and also the quantity of reagents required. Therefore, we designed three multiplex reactions with different primer concentrations: multiplex A $(\mathrm{G} 10 \mathrm{H} 0.8$ $\mathrm{pmol} / \mu \mathrm{l}, \mathrm{G} 1 \mathrm{D} 1 \mathrm{pmol} / \mu \mathrm{l}, \mathrm{G} 10 \times 1 \mathrm{pmol} / \mu \mathrm{l})$, multiplex B $(\mathrm{G} 1 \mathrm{~A} 1 \mathrm{pmol} / \mu 1, \mathrm{G} 10 \mathrm{P} 2$ $\mathrm{pmol} / \mu \mathrm{l}, \mathrm{G} 10 \mathrm{C} 1 \mathrm{pmol} / \mu \mathrm{l})$ and multiplex C (Mu59 $1 \mathrm{pmol} / \mu \mathrm{l}, \mathrm{G} 10 \mathrm{~L} 1 \mathrm{pmol} / \mu 1, \mathrm{Mu} 50$ $1 \mathrm{pmol} / \mu \mathrm{l})$. Only locus Mu26 was amplified in a single reaction. Multiplex PCRs were performed in 10- $\mu 1$ reactions that included $5 \mu \mathrm{l}$ of Qiagen Buffer from the Qiagen multiplex PCR kit, primers and $30 \mathrm{ng}$ of genomic DNA. Thermocycling was performed using an initial step of $95^{\circ} \mathrm{C}$ for $15 \mathrm{~min}$, followed by 35 cycles of $94^{\circ} \mathrm{C}$ for 30 seconds, $57^{\circ} \mathrm{C}$ for $1.5 \mathrm{~min}, 72^{\circ} \mathrm{C}$ for $1 \mathrm{~min}$ with a final extension in $60{ }^{\circ} \mathrm{C}$ for $15 \mathrm{~min}$. Microsatellite loci were labelled in $800 \mathrm{~nm}$ and microsatellite analysis was performed in a semi-automated Li-COR 4200 DNA Analyzer ${ }^{\circledR}$ whereas genotypic data were obtained using SAGA software ${ }^{\circledR}$. 
The multi-tube approach was used in all samples to decrease the probability of genotyping errors. For this reason, two PCRs per locus were initially performed for each sample following the method of Adams and Waits (2007). An allele was accepted only after it was observed twice, otherwise a third PCR was performed and compared with the genotypes of the first two amplifications.

\section{Sex identification}

Sex identification was performed using the primers described by Pages et al. (2009), which co-amplify a bear-specific Y marker ( $S R Y$ gene) and a bear-specific internal PCR control ( $Z F$ gene). PCR amplification was accomplished using the following conditions: initial denaturation at $94^{\circ} \mathrm{C}$ for $5 \mathrm{~min}, 40$ cycles of strand denaturation at $94^{\circ} \mathrm{C}$ for 30 seconds, annealing at $55^{\circ} \mathrm{C}$ for 30 seconds and elongation at $72^{\circ} \mathrm{C}$ for 45 seconds. Final elongation was achieved at $72^{\circ} \mathrm{C}$ for $7 \mathrm{~min}$. Amplifications were performed with a reaction volume of $20 \mu \mathrm{l}$, containing $2 \mu \mathrm{l}$ of $10 \times$ Reaction Buffer, $0.2 \mu \mathrm{l}$ of $10 \times$ bovine serum albumin, $0.25 \mathrm{mM}$ dNTPs, $1 \mathrm{pmol} / \mu 1$ of each SRY primer, $6 \mathrm{pmol} / \mu \mathrm{l}$ of each ZF primer and approximately $50-100 \mathrm{ng}$ of template DNA. Visualization and control of the PCR products were achieved by electrophoresis in $1.5 \%$ agarose gel.

\section{Statistical analysis}

\section{Genotyping reliability - markers suitability}

DROPOUT software (McKelvey and Schwartz 2005) was used to determine whether a sample contained genotyping errors, the relative magnitude of the problem as well as the number of unique genotypes. For all the genotypes for which a discrepancy was detected between the first and the second PCR amplification the program RELIOTYPE (Miller et al. 2002) was used to detect if the reliability level of $95 \%$ was reached after the third PCR amplification (the minimum acceptable level). In the final data set, we retained only genotypes with average reliability scores $\geq 95 \%$.

To evaluate the suitability of the marker set for identifying individuals, the probability of identity $\left(P_{\mathrm{ID}}\right.$; Paetkau and Strobeck 1994) and the more conservative probability of identity among siblings ( $P_{\mathrm{ID}-\mathrm{Sib}}$; Waits et al. 2001) were estimated using the software GIMLET v. 1.3.2 (Valiere 2002).

\section{Genetic diversity and inbreeding}

Observed $\left(H_{\mathrm{o}}\right)$ and expected $\left(H_{\mathrm{e}}\right)$ heterozygosity values for each locus were calculated using GENEPOP 4.0 (Rousset 2008). Deviation from Hardy-Weinberg equilibrium was tested using Fisher's exact tests (Rousset 2008) with unbiased $p$-values derived by a Markov chain method with the same software. The significance value for multiple significance tests was set using the sequential Bonferroni procedure (Rice 1989). CERVUS 3.0.3 (Kalinowski et al. 2007) was used to evaluate polymorphic information content, null allele probability and number of alleles for each locus. 


\section{Population structure and demographic history}

The numbers of genetic clusters $(K)$ was inferred using STRUCTURE 2.3.4 (Pritchard et al. 2000; Falush et al. 2007). The method uses a Bayesian clustering algorithm to partition individuals into a given number of populations $(K)$ under the assumption of Hardy-Weinberg equilibrium and linkage equilibrium. The admixture model was used, allele frequencies were assumed to be independent and analyses were conducted with a burn-in period of 50,000 followed by 750,000 Markov chain Monte Carlo repetitions. We ran STRUCTURE setting the number of clusters $(K)$ from 1 to 5 (with 10 runs for each $K$ ) to determine the most likely number of clusters representative of the data. The most probable value of $K$ was inferred from the mean loglikelihood of the $10 \times[\operatorname{LnP}(\mathrm{D})]$ values according to the criteria by Pritchard et al. (2000). $K$ with the highest likelihood and consistency between runs was chosen as the most appropriate.

A factorial correspondence analysis implemented in the program GENETIX v. 4.05.2 (Belkhir et al.1996, 2004) was performed to graphically visualize the genetic relationship between individuals and inferred groups. In addition, an exclusion test (Cornuet and Luikart 1996) for detecting potential immigrants in the population was performed using the software GENECLASS v. 2.0 (Piry et al. 2004), applying the frequency-based method (Paetkau et al. 1995) and the simulation algorithm of Paetkau et al. (2004).

To test for recent genetic bottlenecks, deviations from expected heterozygosity were inferred under the assumption of mutation drift equilibrium by either a stepwise mutation model or a two-phase model using the program BOTTLENECK 1.2.02 (Cornuet and Luikart 1996). The data were analysed with the recommended settings (Piry et al. 1999).

\section{Estimation of census $\left(N_{c}\right)$ and effective $\left(N_{e}\right)$ population size}

Census population size was estimated using the estimator implemented in the capture-mark-recapture-based programme for NGS CAPWIRE (Miller et al. 2005). In traditional trap-based mark-recapture studies, an individual may be captured only once per session. Estimating population size has focused on estimating the probability of capture for each individual in each session. An important difference in the data arising from DNA-based mark-recapture studies is that sampling is approximately done with replacement. That is, since an individual is not physically confined at any time, it may leave multiple hair tufts or scats at multiple locations during a sampling session. CAPWIRE accommodates data with multiple observations of an individual within a single session and has been shown to work well with capture heterogeneity and small populations ( $<100$ individuals), such as the one expected in our study area (Miller et al. 2005). Possible capture heterogeneity in our data, due to the collection of genetic samples from power poles (Karamanlidis et al. 2007, 2010), necessitated the use of the two innate rates model for the calculation of population size.

The effective population size $N_{e}$ of the brown bear population was estimated using one-point estimate methodologies implemented in NeESTIMATOR 1.3 software (Peel et al. 2004). $N_{e}$ was calculated using the linkage disequilibrium method option of NeESTIMATOR. Results were also compared with the one-point $N_{e}$ estimate given by ONeSAMP 1.2 (Tallmon et al. 2008). This software uses 
summary statistics and approximate Bayesian computation to estimate $N_{e}$ from a single sample.

\section{Results}

\section{Individuals and sex identification}

The total number of samples collected in the field during the whole sampling season was 232 (171 hair samples, 46 scat samples and 15 blood samples). However, 18 hair samples had no hair root and they were not included in the analysis. DNA was successfully extracted from 116 hair samples (76\%), 22 faecal samples (48\%) and 12 blood samples $(80 \%)$. The quality of the hair root was very important for the successful DNA extraction; in more than 10 cases the use of only two or three high-quality hair roots was sufficient to obtain amplification in all the selected microsatellite loci.

The multi-tube PCR approach was used for hair root and faecal sample amplification to decrease genotyping error. Furthermore, multiplex PCR procedures, which co-amplify several loci in the same reaction and so decrease workload and cost, and enable a more efficient use of the template DNA (Skrbinsěk et al. 2010), were applied. Hence, from the successfully extracted DNA samples, 86 hair, 20 faecal and 12 blood samples were fully genotyped for the 10 loci and they were used for further analysis. From the 1060 genotypes obtained in non-invasive samples, only 28 (2.6\%) differed in the alleles between the first and second PCR and so a third PCR was performed. In the final data set, 16 genotypes were rejected because they retained average reliability scores $<95 \%$.

In total, 82 unique genotypes were identified representing 82 different bears. No mismatches were recorded when analysing blood and hair or faecal samples from the same individual. Sex identification was achieved in 72 individuals and the majority of them (48 individuals) were males. The male : female ratio was $2: 1$.

\section{Genetic diversity}

All loci in the study were polymorphic, with the number of alleles per locus ranging between 3 and 10 and a mean of 5.8 (Table 1). Mu59 was the most variable locus with an observed heterozygosity value of 0.771 , whereas G10X was the least polymorphic with an observed heterozygosity value of 0.085 . More than $70 \%$ of the selected markers had high polymorphic information content, showing how informative these markers were in evaluating genetic diversity (Table 1). The probability of identity among siblings $\left(P_{\mathrm{ID}-\mathrm{Sib}}\right)$ was $<0.01$, recommending that the data can be used for population size estimation (Waits et al. 2001).

Concerning Hardy-Weinberg tests per locus, only G10P and Mu26 loci show deviation from equilibrium at a nominal level of $5 \%$, possibly due to null alleles $(F=0.146$ and 0.124 , respectively, Table 1$)$. The population showed deviation from the Hardy-Weinberg equilibrium, but when the G10P locus was excluded from the analysis, the population was in equilibrium $(P=0.089)$. The inbreeding coefficient value over all loci was very low $\left(F_{\text {IS }}=0.07\right)$ indicating a lack of heterozygosity deficiency. The mean observed heterozygosity was 0.584 and the expected was 0.548 . 
Table 1. Number of alleles $(A)$, allelic size range in base pairs $(R)$, observed and expected heterozygosity $\left(H_{\mathrm{o}}, H_{\mathrm{e}}\right)$, probability value for Hardy-Weinberg tests $\left(P_{\mathrm{HW}}\right)$, polymorphic information content (PIC), null alleles per locus $(F)$, probability of identity $\left(P_{\text {ID }}\right)$ and the probability of identity among siblings $\left(P_{\mathrm{ID}-\mathrm{Sib}}\right)$ for the Kastoria brown bear population.

\begin{tabular}{lcrccccccc}
\hline Locus & $A$ & $R$ & $H_{\mathrm{o}}$ & $H_{\mathrm{e}}$ & $P_{H W}$ & PIC & \multicolumn{1}{c}{$F$} & $P_{I D}$ & $P_{I D-S i b}$ \\
\hline G10C & 6 & $97-107$ & 0.728 & 0.766 & 0.480 & 0.722 & 0.021 & $8.77 \mathrm{E}-02$ & $3.85 \mathrm{E}-01$ \\
G10P & 5 & $137-157$ & 0.513 & 0.700 & 0.004 & 0.642 & 0.146 & $7.23 \mathrm{E}-03$ & $1.49 \mathrm{E}-01$ \\
G1A & 5 & $180-194$ & 0.720 & 0.635 & 0.400 & 0.574 & -0.079 & $6.97 \mathrm{E}-04$ & $5.86 \mathrm{E}-02$ \\
G10X & 3 & $134-152$ & 0.085 & 0.083 & 1.000 & 0.080 & -0.012 & $6.61 \mathrm{E}-05$ & $2.32 \mathrm{E}-02$ \\
G1D & 6 & $171-183$ & 0.709 & 0.762 & 0.287 & 0.722 & 0.029 & $9.65 \mathrm{E}-06$ & $1.02 \mathrm{E}-02$ \\
G10H & 6 & $226-260$ & 0.573 & 0.588 & 0.054 & 0.524 & 0.009 & $1.86 \mathrm{E}-06$ & $4.90 \mathrm{E}-03$ \\
G10L & 5 & $146-166$ & 0.402 & 0.422 & 0.114 & 0.398 & 0.007 & $4.35 \mathrm{E}-07$ & $2.53 \mathrm{E}-03$ \\
Mu50 & 6 & $100-110$ & 0.765 & 0.771 & 0.337 & 0.737 & -0.000 & $1.56 \mathrm{E}-07$ & $1.60 \mathrm{E}-03$ \\
Mu59 & 10 & $220-252$ & 0.744 & 0.773 & 0.937 & 0.739 & 0.019 & $7.29 \mathrm{E}-08$ & $1.12 \mathrm{E}-03$ \\
Mu26 & 6 & $180-200$ & 0.244 & 0.331 & 0.031 & 0.311 & 0.124 & $6.15 \mathrm{E}-08$ & $1.03 \mathrm{E}-03$ \\
Mean & 5.8 & & 0.584 & 0.548 & 0.009 & 0.545 & & & \\
\hline
\end{tabular}

\section{Genetic structure and demographic history}

STRUCTURE analysis results (Figure 2a) provided the strongest support for the partitioning of the genetic variation into only one cluster, indicating that the bear population in the Kastoria region shows no sign of substructure and it can be regarded as panmictic. This result was also supported by factorial correspondence analysis (Figure 2b), as only one cluster of individuals was identified. One individual most likely did not originate from the sampled population (exclusion test, $P<0.0001$ ) and was probably an emigrant.

Tests for bottleneck phenomena were not significant for both stepwise and two mutation phase models for any sample $(0.306<\mathrm{P}<0.13$ and $0.2<\mathrm{P}<0.4$, respectively) and showed normal L-shaped distributions in mode shift. Hence, the analysis showed no evidence of a recent genetic bottleneck.

\section{Census and effective population size}

In total, 82 individuals (Ursus1-Ursus82) were identified according to their composite genotype. Sixty-nine individuals were detected from hair or faecal samples, while six individuals were detected from blood samples taken from living bears that were caught for radio-tracking or survived from vehicle collisions. The remaining seven individuals were victims of collisions and so they were excluded from the living population. Therefore, the minimum population size of bears in the Kastoria region during 2011 was according to our results 75 individuals. The sampling locations of all living bears identified in this study are presented in Figure 1. To estimate the effective $\left(N_{e}\right)$ and the census $(N)$ population size only these 75 living individuals were used.

The number of "captures" per individual ranged from one to six. Most of the individuals (58) were "captured" only once but 17 bears were "recaptured" once or more. Details about the number of, date of and maximum distance between recaptures are given in Table 2. For the majority of the cases, the "recapture" samples were 

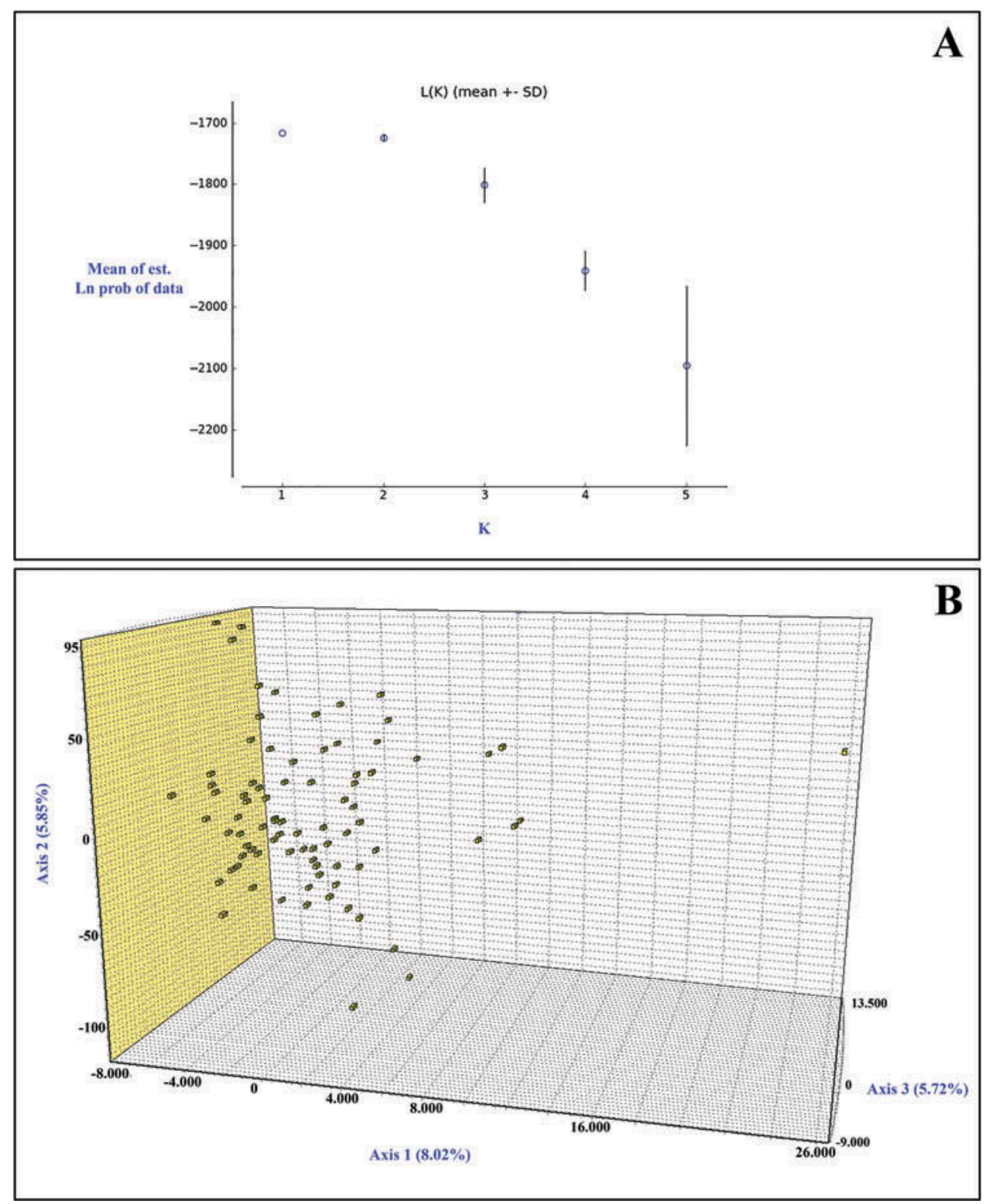

Figure 2. (A) Means of estimated LnP (Data) and standard deviations for $K=1$ to $K=5$. (B) Factorial correspondence analysis plot of multilocus genotypes for 82 brown bear individuals identified in the present study.

collected at different locations but within small distances (maximum distance: $12 \mathrm{~km}$ ). The sex ratio for the individuals that were recaptured was 11 males $/ 6$ females.

The analysis with program CAPWIRE, which is based on the number of captures and recaptures (Table 2), assuming that all samples have been collected in a single sampling session, resulted in a point estimation for $N_{c}$ of 219 individuals. The $95 \%$ confidence interval $(95 \% \mathrm{CI})$ was $145-271$ individuals; 17 individuals were classified 


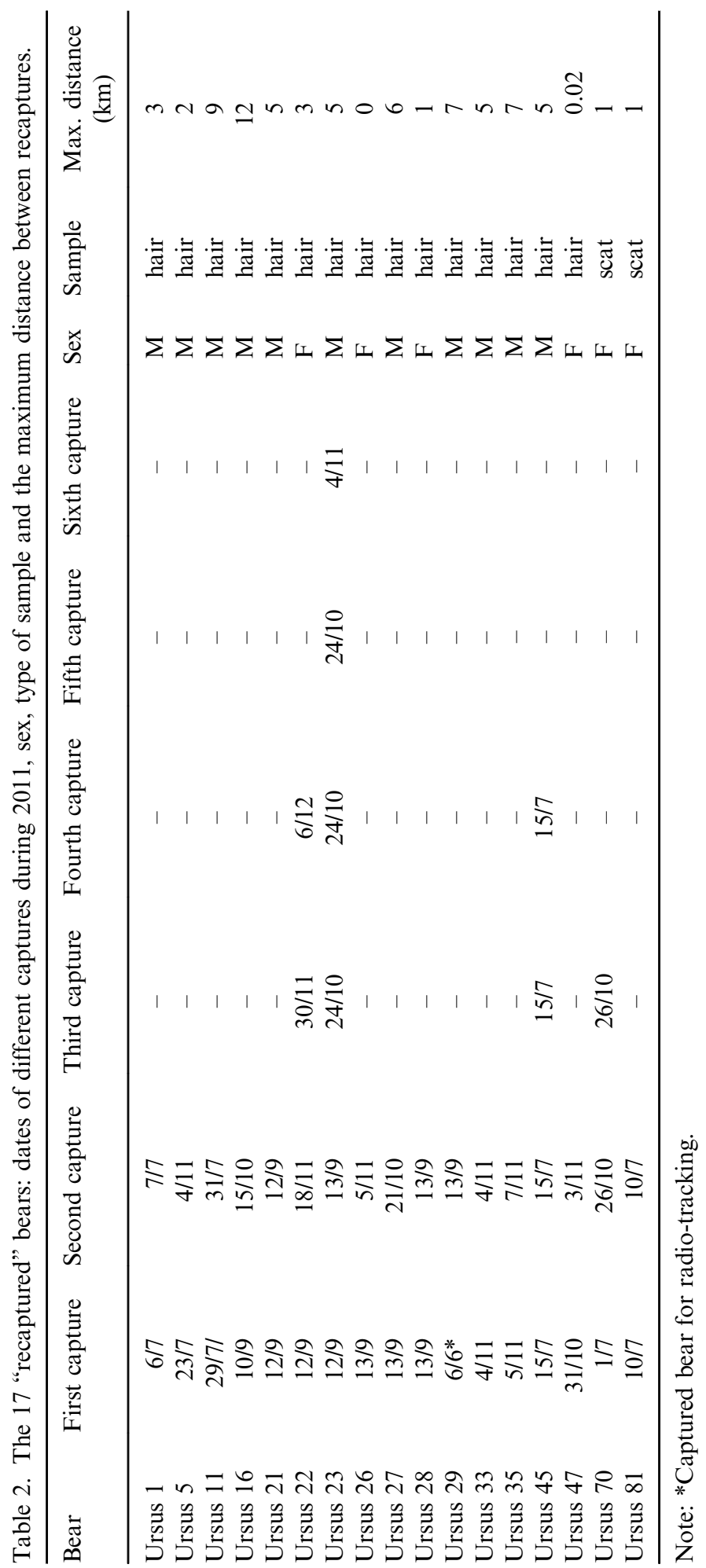


as easier to capture (type A), and the remaining 202 as harder to capture (type B). Point estimates of $N_{e}$ using NeESTIMATOR gave a mean value of 48.7 (95\% CI 37.1-65.1). When applying the OneSAMP software, values of $N_{e}$ were somewhat lower: 39.5 (95\% CI 29.2-65.3).

\section{Discussion}

\section{Reliability of NGS and sampling procedure}

Non-invasive sampling of hair and faeces (as performed in our study) proved to be an efficient method for obtaining adequate genetic data on the brown bear population in the Kastoria region, without handling or disturbing the animals. The choice of a combined sampling strategy (mainly systematic hair sampling but also opportunistic faecal sampling) allowed us to collect a sufficient number of samples providing a larger number of identified bears. In fact, the collection of 46 faecal samples allowed the identification of 17 more bears, which otherwise would have been excluded from the analysis. From a total of 217 non-invasively collected samples, we managed to fully genotype 106, resulting in an almost 50\% total rate of success. These rates are within the range of values reported in other non-invasive genetic studies of bears and it can be considered acceptable in terms of cost effectiveness (Kohn et al. 1999; Solberg et al. 2006; Pérez et al. 2009; Sawaya et al. 2012).

A major concern about NGS is the accuracy of results because genotyping of samples collected in this way is prone to genotyping errors (allelic dropout, false alleles) (Waits and Paetkau 2005). Genotyping errors can inflate the number of identified individuals in a data set, so checking for genotyping accuracy is necessary to avoid misleading results. Implementation of the multi-tube approach was chosen as an appropriate method for controlling genotyping errors (Pompanon et al. 2005). Consistency of genotypes in both PCRs for the vast majority of samples and final rejection of very few genotypes $(1.5 \%)$ with average reliability scores $<95 \%$ strongly support the reliability of bear identifications in our study.

High success rates of DNA extraction and amplification, low rates of genotyping errors and lack of mismatches between blood and non-invasive samples from the same individual confirm the reliability of the NGS methodology that can provide good-quality data. However, specific considerations for the sampling procedure that could improve the quantity of the collected data in future surveys would be useful to report at this point. The sampling period for hair trapping in our study lasted from midsummer till early winter. According to Karamanlidis et al. (2010) the best period for collecting hair from the power poles is late spring and early summer because of the intense marking and rubbing behaviour of brown bears during the mating season. However, specific technical constraints hampered the beginning of our sampling at this period. A better description of bear distribution and possible identification of seasonal movements are expected in the forthcoming sampling sessions, by taking advantage of the installed permanent hairtrap network for collecting hair samples throughout the year. In addition, the collection of more samples will provide more solid statistical analysis and more accurate estimations of population size.

One of the main drawbacks of using hairtraps on power poles for collecting hair samples is that the availability and geographical distribution of power poles determine the sampling scheme and capture probabilities that may lead to uneven 
sampling intensity. In our study, most of the collected hair samples, and consequently most of the identified bears, were located in the central part of the Kastoria prefectural unit (see Figure 1) whereas very few samples were collected in the neighbouring mountainous regions. In the remote forested areas a dense power pole network does not usually exist. This fact hampered the establishment of an evenly distributed sampling network in our study area. Opportunistic sampling of faeces was used to overcome the problem, although the number of suitable faecal samples collected was not large enough for a detailed description of bear distribution throughout the study area. Hence, extensive scat sampling, baited hairtraps (Sawaya et al. 2012) or haitraps on rub trees (Woods et al. 1999) could be proper - albeit more laborious - alternatives for collecting more genetic samples in those areas where the power pole network is sparse.

\section{Moderate levels of diversity and lack of genetic structure}

The bear population of Kastoria exhibits medium to high levels of nuclear genetic diversity, lower than that reported for large populations of north Europe, Romania or Russia but higher in comparison with some small populations in central and southern Europe (see Swenson et al. 2011). Isolated, endangered bear populations in Spain and Italy for instance that have suffered severe historical demographic bottlenecks show low values of observed heterozygosity $\left(H_{\mathrm{o}}=0.28-0.44\right)$ and very few alleles per locus $(A=1.7-3.3)$ (Lorenzini et al. 2004; Pérez et al. 2009). In contrast, the Kastoria bear population seems to retain significant levels of genetic diversity $\left(H_{\mathrm{o}}=0.58, A=5.8\right)$, and our analysis with BOTTLENECK software failed to detect a recent bottleneck event. Although there is no information about the demographic history of bears in Kastoria, the possibility of an older or not severe bottleneck (not detectable by the software) and subsequent population recovery of the local population cannot be excluded.

No evidence of substructure or heterozygosity deficiency was detected, indicating the existence of a panmictic population that faces a low risk of inbreeding. The KA45 highway disrupts the bear habitat in Kastoria but it is too early to test whether this acts as an effective barrier because it has only been in operation for 3 years. Preliminary telemetry data on bear spatial behaviour over a sample of six bears monitored in the study area in 2011 show that home ranges do not seem, at least at this stage, to be confined by the highway (Mertzanis and Iliopoulos 2011). Genetic recaptures of some individuals on both sides of the highway during this study confirm the occasional permeability of the KA45 highway. Especially for the part of the highway between Dryovouno and Vogatsiko villages, the frequency of crossings by bears seems to be significantly higher (Mertzanis and Iliopoulos 2011). Nine bears were "captured" at hairtraps attached on the highway fence of this section during a period of less than 2 months. Four of them were females, probably accompanied by cubs.

Effective population size $\left(N_{e}\right)$ estimated in our study (40-49 individuals, depending on the analysis), was close enough to the minimum threshold of 50 individuals, which is supposed to be adequate for avoiding inbreeding depression (Frankham et al. 2002). Hence, relatively high diversity values, lack of heterozygosity deficiency and the $N_{e}$ values, support the Kastoria bear population being in good conservation status and at least within the near future, it will not be at risk of genetic depletion. 
Kastoria region is part of the bear distribution range of northwestern Greece which encompasses the Pindos mountain range and the attached massifs. A bear population in central Pindos (Grevena region) has also been studied by Karamanlidis et al. (2012). Similar genetic diversity values $\left(H_{\mathrm{o}}=0.65, A=5.6\right)$, and a lack of substructuring were also reported. Significant levels of ongoing gene flow between contiguous populations of the Pindos range (e.g. Tymfi, Smolikas, Grammos) and the attached massifs like Askio or Vernon into a meta-population concept (Hanski 1999) could be a possible explanation for the significant values of genetic diversity in the Kastoria and Grevena populations. In addition, close proximity to bear populations of southern Albania and FYROM probably allows a permanent connection between them and sufficient levels of gene flow. A genetic study of Greek bears at a greater geographic scale could delineate the population structure and reveal the connectivity patterns between the main regions of the current bear distribution.

\section{Estimated population size and suggestions for improving accuracy}

One of the main goals of this study was estimation of the size of the brown bear population in Kastoria. The NGS and the subsequent amplification for 10 microsatellite loci allowed the identification of 75 living bears in the region, indicating the presence of a minimum population of this size. Most of the identified bears were males (twice as many as females) possibly because of heterogeneity in capture probability. Rubbing and marking behaviour on power poles has been reported to be more intense among male bears than females, leading to unequal hair sample collection between sexes (Green and Mattson 2003; Karamanlidis et al. 2010). More intensive scat sampling in future surveys could help to overcome a possible underestimation of female bears.

Analysis with the CAPWIRE program gave a point estimation significantly higher (about three times) than the minimum population of identified bears. Noninvasive DNA-based $N_{c}$ estimates with this method can be obtained from a single sampling session. The ability to estimate $N_{c}$ from a single sampling session is extremely helpful for species that are costly or time-consuming to sample. However, a major concern and a basic assumption in most DNA-based markrecapture models, including the CAPWIRE "urn" model, is population closure (Boulanger and McLellan 2001; Miller et al. 2005). The population closure assumption rests on there being no immigration, emigration, birth or death. No births occurred during our sampling period because bears give birth in late winter. In addition, to avoid positive bias in census population size estimation, we excluded from analysis those samples that were taken from dead bears (victims of vehicle collisions). On the other hand, we cannot exclude the possibility of bear deaths due to illegal hunting or poisoning in the study area during our sampling period because in most cases such incidents happen in remote forested areas and usually they remain unrevealed. The existence of at least one individual that seems not to belong to the local population, according to our results of exclusion tests and factorial correspondence analysis, indicates that emigration is another possibility that violated closure assumption.

Significant closure violation - resulting in a positive bias in population estimates - can also occur when animal home ranges are relatively large and exceed the sampling area (Boulanger and McLellan 2001). Movements of bears in and out of 
the sampling area can inflate the number of "captured" animals and negatively bias "recapture" probability. In these cases, the estimated with closed-model methods population size most probably describes the "superpopulation" of animals in the predefined sampling area but in the surrounding areas as well (Kendall 1999). According to telemetry data from six radio-collared bears in the study area during 2011, male bears have large home ranges (165-226 $\left.\mathrm{km}^{2}\right)$ whereas females' ranges are significantly smaller (10-91 km²) (Mertzanis and Iliopoulos 2011). Considering the continuous bear habitats in the surrounding areas as well as the fact that the mean recapture rate of 1.36 observations/individual was significantly lower than the 2-3 observations/individual recommended by Miller et al. (2005) we should not exclude the possibility of positive bias in our estimation.Hence, the estimated census size should be treated with caution, especially for density estimates, because it rather corresponds to a "superpopulation" inhabiting an area larger than the Kastoria prefectural unit. Repetition of the survey in the forthcoming years using the proposed improvements is expected to give more accurate estimates. In addition, extensive and simultaneous telemetry is recommended to index movements of bears in and out of the study area (Powell et al. 2000).

The bear population of Kastoria region may be considered among the most robust bear populations in Greece. The identification of 75 bears in the study area during the present study is enough to highlight the significant abundance of this population. The minimum population size of 75 bears corresponds approximately to $30 \%$ of the minimum bear population size estimated for the whole of Greece by Mertzanis et al. (2009a). High abundance in conjunction with significant levels of genetic diversity and good conservation status make the Kastoria bear population a significant stock for conservation of the species in Greece. Long-term monitoring and management actions to maintain sufficient levels of gene flow must be of high priority.

\section{Acknowledgements}

This study was financed and became possible under project LIFE09NAT/GR/000333 (DGENV), with Coordinating Beneficiary the Region of Western Macedonia, and NGO "Callisto" as the key partners. We would like to thank Giannis Tsaknakis, Armin and Sussane Riegler, Thanos Tragos, George Lazarou and Nick van Dormaal for their support in sampling and Ilias Aravidis for his assistance in the GIS data processing and mapping. This article is in memory of our dearest colleague Konstantinos Godes.

\section{References}

Adams JR, Waits LP. 2007. An efficient method for screening faecal DNA genotypes and detecting new individuals and hybrids in the red wolf (Canis rufus) experimental population area. Conserv Genet. 8:123-131.

Belkhir, et al. 1996-2004. GENETIX 4.05, logiciel sous Windows TM pour la génétique des populations.

Bellemain E, Swenson JE, Tallmon D, Brunberg S, Taberlet P. 2005. Estimating population size of elusive animals using DNA from hunter-collected feces: comparing four methods for brown bears. Conserv Biol. 19:150-161.

Bellemain E, Taberlet P. 2004. Improved noninvasive genotyping method: application to brown bear (Ursus arctos) faeces. Mol Ecol Notes. 4:519-522. 
Boulanger J, McLellan B. 2001. Closure violation in DNA-based mark-recapture estimation of grizzly bear populations. Can J Zoolog. 79:642-651.

Cornuet JM, Luikart G. 1996. Description and power analysis of two tests for detecting recent population bottlenecks from allele frequency data. Genetics. 144:2001-2014.

Creel S, Spong G, Sands JL, Rotella J, Zeigle J, Joe L, Murphy KM, Smith D. 2003. Population size estimation in Yellowstone wolves with error-prone noninvasive microsatellite genotypes. Mol Ecol. 12:2003-2009.

De Barba M, Waits LP, Garton EO, Genovesi P, Randi E, Mustoni A, Groff C. 2010a. The power of genetic monitoring for studying demography, ecology and genetics of a reintroduced brown bear population. Mol Ecol. 19:3938-3951.

De Barba M, Waits LP, Genovesi P, Randi E, Chirichella R, Cetto E. 2010b. Comparing opportunistic and systematic sampling methods for non-invasive genetic monitoring of a small translocated brown bear population. J Appl Ecol. 47:172-181.

Falush D, Stephens M, Pritchard JK. 2007. Inference of population structure using multilocus genotype data: dominant markers and null alleles. Mol Ecol Notes. 7:574-578.

Frankham R, Ballou JD, Briscoe DA. 2002. Introduction to conservation genetics. Cambridge: Cambridge University Press.

Gagneux P, Boesch C, Woodruff DS. 1997. Microsatellite scoring errors associated with noninvasive genotyping based on nuclear DNA amplified from shed hair. Mol Ecol. 6:861-868.

Gervasi V, Ciucci P, Boulanger J, Posillico M, Sulli C, Focardi S, Randi E, Boitani L. 2008. A preliminary estimate of the Apennine brown bear population size based on hair-snag sampling and multiple data source mark-recapture Huggings models. Ursus. 19:105-121.

Green GI, Mattson DJ. 2003. Tree rubbing by Yellowstone grizzly bears Ursus arctos. Wildlife Biol. 9:1-9.

Hanski I. 1999. Metapopulation Ecology. Oxford: Oxford University Press.

Kalinowski ST, Taper ML, Marshall TC. 2007. Revising how the computer program CERVUS accommodates genotyping error increases success in paternity assignment. Mol Ecol. 16:1099-1106.

Karamanlidis AA. 2008. Development of an innovative method for studying genetic, demographic and behavioural aspects of the brown bear (Ursus arctos) [ $\mathrm{PhD}$ thesis]. Thesssaloniki (GR): Aristotle University of Thessaloniki.

Karamanlidis AA. 2011. First Genetic Census of brown bear (Ursus arctos) population in Greece. Final Report; Arcturos, Hellenic Ministry of Rural Development and Food \& Hellenic Ministry of Environment, Energy and Climate Change; Thessaloniki; pp. 1-87 greek.

Karamanlidis AA, Drosopoulou E, De Gabriel Hernando M, Georgiadis L, Krambokoukis L, Pllaha S, Zedrosser A, Scouras Z. 2010. Noninvasive genetic studies of brown bears using power poles. Eur J Wildlife Res. 56:693-702.

Karamanlidis AA, Straka M, Drosopoulou E, De Gabriel Hernando M, Kocijan I, Paule L, Scouras Z. 2012. Genetic diversity, structure, and size of an endangered brown bear population threatened by highway construction in the Pindos Mountains, Greece. Eur $\mathbf{J}$ Wildlife Res. 58:511-522.

Karamanlidis AA, Youlatos D, Sgardelis S, Scouras Z. 2007. Using sign at power poles to document presence of bears in Greece. Ursus. 18:54-61.

Kendall WL. 1999. Robustness of closed capture-recapture methods to violations of the closure assumption. Ecology. 80:2517-2525.

Kohn MH, Wayne RK. 1997. Facts from feces revisited. Trends Ecol Evol. 12:223-227.

Kohn MH, York EC, Kamradt DA, Haught G, Sauvajot RM, Wayne RK. 1999. Estimating population size by genotyping feces. P Roy Soc Lond B. 266:657-663.

Lathuillière M, Ménard N, Gautier-Hion A, Crouau-Roy B. 2001. Testing the reliability of noninvasive genetic sampling by comparing analyses of blood and fecal samples in Barbary Macaques (Macaca sylvanus). Am J Primatol. 55:151-158. 
Linnell J, Salavatori V, Boitani L. 2007. Guidelines for population level Management Plans for large carnivores in Europe. LCIE Techical report, EC Project 070501/2005/424162/MAR/ B2. 78pp.

Lorenzini R, Posillico M, Lovari S, Petrella A. 2004. Non-invasive genotyping of the endangered Apennine brown bear: a case study not to let one's hair down. Anim Conserv. 7:199-209.

Luikart G, Ryman N, Tallmon DA, Schwartz MK, Allendorf FW. 2010. Estimation of census and effective population sizes: the increasing usefulness of DNA-based approaches. Conserv Genet. 11:355-373.

McKelvey KS, Schwartz MK. 2004. Genetic errors associated with population estimation using non-invasive molecular tagging: problems and new solutions. J Wildlife Manage. 68:439-448.

McKelvey K, Schwartz MK. 2005. DROPOUT: a program to identify problem loci and samples for noninvasive genetic samples in a capture-mark-recapture framework. Mol Ecol Notes. 5:716-718.

Mertzanis G. 1994. Brown bear in Greece: distribution, present status-ecology of a northern Pindus subpopulation. Proc Int Conf Bear Res Manage. 9:187-197.

Mertzanis G, Giannakopoulos A, Pilides C. 2009a. Status of the brown bear Ursus arctos (Linnaeus, 1758) in Greece. p. 385-387 In: Legakis \& Maragou P, editors.. Red data book of threatened vertebrates of Greece. Athens: Hellenic Zoological Society. 525 pp.

Mertzanis G, Iliopoulos G. 2011. Identification and effective delineation of sectors over the main road and highway network with high risk of bear (Ursus arctos) traffic accidents for minimization of bear related mortality. Callisto NGO, Thessaloniki. Project LIFE09NAT/GR/00333; Technical report; 84pp.

Mertzanis G, Korakis G, Tsiokanos K, Aravidis I. 2009b. Expansion of brown bear range in the course of rural abandonment during the 20th century - a case study from the Pindos mountain range. p. 330-337. In: Saratsi E, Burgi M, Johann E, Kirby K, Moreno D, Watkins C, editors. Woodland cultures in time and space - tales from the past, messages for the future. Athens: Embryo; $400 \mathrm{pp}$.

Miller C, Joyce P, Waits L. 2002. Assessing allelic dropout and genotype reliability using maximum likelihood. Genetics. 160:357-366.

Miller CR, Joyce P, Waits LP. 2005. A new method for estimating the size of small populations from genetic mark-recapture data. Mol Ecol. 14:1991-2005.

Paetkau D, Calvert W, Stirling I, Strobeck C. 1995. Microsatellite analysis of population structure in Canadian polar bears. Mol Ecol. 4:347-354.

Paetkau D, Shields GF, Strobeck C. 1998. Gene flow between insular, coastal and interiorpopulations of brown bears in Alaska. Mol Ecol. 7:1283-1292.

Paetkau D, Slade R, Burden M, Estoup A. 2004. Genetic assignment methods for the direct, real-time estimation of migration rate: a simulation-based exploration of accuracy and power. Mol Ecol. 13: 55-65.

Paetkau D, Strobeck C. 1994. Microsatellite analysis of genetic variation in black bear populations. Mol Ecol. 3:489-495.

Pagès M, Maudet C, Bellemain E, Taberlet P, Hughes S, Hänni C. 2009. A system for sex determination from degraded DNA: a useful tool for palaeogenetics and conservation genetics of ursids. Conserv Genet. 10:897-907.

Peel D, Ovenden JR, Peel SL. 2004. NeESTIMATOR: Software for estimating effective population size, version 1.3. Brisbane (Queensland): Queensland Government, Department of Primary Industries and Fisheries.

Pérez T, Vazquez F, Naves J, Fernandez A, Corao A, Albornoz J, Dominguez A. 2009. Noninvasive genetic study of the endangered Cantabrian brown bear (Ursus arctos). Conserv Genet. 10:291-301.

Piry S, Alapetite A, Cornuet J-M, Paetkau D, Baudouin L, Estoup A. 2004. GENECLASS2: a software for genetic assignment and first-generation migrant detection. J Hered. 95:536-539. 
Piry S, Luikart GL, Cornuet J-M. 1999. BOTTLENECK: a computer program for detecting recent reductions in the effective size using allele frequency data. J Hered. 90:502-503.

Pompanon F, Bonin A, Bellemain E, Taberlet P. 2005. Genotyping errors: causes consequences, and solutions. Nat Rev Genet. 6:847-859.

Powell LA, Conroy M, Hines JE, Krementz DG. 2000. Simultaneous use of mark-recapture and radiotelemetry to estimate survival, movement, and capture rates. $\mathrm{J}$ Wild Manage. 64:302-313.

Pritchard JK, Stephens M, Donnelly PJ. 2000. Inference of population structure using multilocus genotype data. Genetics. 155:945-959.

Rice WR. 1989. Analyzing tables of statistical tests. Evolution. 43:223-225.

Rousset F. 2008. GENEPOP'007: a complete re-implementation of the GENEPOP software for Windows and Linux. Mol Ecol Resour. 8:103-106.

Sawaya MA, Ruth TK, Creel S, Rotella JJ, Stetz JB, Stetz JB, Quigley HB, Kalinowski ST. 2011. Evaluation of noninvasive genetic sampling methods for cougars in Yellowstone National Park. J Wild Manage. 75:612-622.

Sawaya MA, Stetz JB, Clevenger AP, Gibeau ML, Kalinowski ST. 2012. Estimating grizzly and black bear population abundance and trend in Banff National Park using noninvasive genetic sampling. Plos One. 7:1-12.

Schregel U, Kopatz A, Hagen SB, Brøseth H, Smith M, Wikan S, Wartiainen I, Aspholm PE, Aspi J, Swenson J, et al. 2012. Limited gene flow among brown bear populations in far Northern Europe? Genetic analysis of the east-west border population in the Pasvik Valley. Mol Ecol. 21:3474-3488.

Skrbinsěk T, Jelenčič M, Waits L, Kos I, Trontelj P. 2010. Highly efficient multiplex PCR of noninvasive DNA does not require pre-amplification. Mol Ecol Resour. 1:495-501.

Solberg KH, Bellemain E, Drageset OM, Taberlet P, Swenson JE. 2006. An evaluation of field and non-invasive genetic methods to estimate brown bear (Ursus arctos) population size. Biol Conserv. 128:158-168.

Soulé ME. 1987. Viable populations for conservation. Cambridge: Cambridge University Press.

Stenglein JL, de Barba M, Ausband DE, Waits LP. 2010. Impacts of sampling location within a faeces on DNA quality in two carnivore species. Mol Ecol Res. 10:109-114.

Swenson JE, Taberlet P, Bellemain E. 2011. Genetics and conservation of European brown bears Ursus arctos. Mammal Rev. 41:87-98.

Taberlet P, Camarra JJ, Griffin S, Uhres E, Hanotte O, Waits LP, Dubois-Paganon C, Burke T, Bouvet J. 1997. Noninvasive genetic tracking of the endangered Pyrenean brown bear population. Mol Ecol. 6:869-876.

Taberlet P, Griffin S, Goossens B, Questiau S, Manceau V, Escaravage N, Waits L, Bouvet J. 1996. Reliable genotyping of samples with very low DNA quantities using PCR. Nucleic Acids Res. 24:3189-3194.

Tallmon DA, Koyuk A, Luikart GH, Beaumont MA. 2008. ONeSAMP: a program to estimate effective population size using approximate Bayesian computation. Mol Ecol Resour. 8:299-301.

Valiere N. 2002. GIMLET: a computer program for analysing genetic individual identification data. Mol Ecol Notes. 2:377-379.

Waits LP, Luikart G, Taberlet P. 2001. Estimating the probability of identity among genotypes in natural populations: cautions and guidelines. Mol Ecol. 10:249-256.

Waits LP, Paetkau D. 2005. Noninvasive genetic sampling tools for wildlife biologists: a review of applications and recommendations for accurate data collection. J Wildlife Manage. 69:1419-1433.

Woods JG, Paetkau D, Lewis D, McLellan BN, Proctor M, Strobeck C. 1999. Genetic tagging of free-ranging black and brown bears. Wildlife Soc B. 27:616-627. 